\title{
Molecular Fingerprinting for Detecting Genetic Relationships among different Accessions of Pterocarpus marsupium
}

\author{
Anees Ahmad ${ }^{1}$, Naseem Ahmad ${ }^{1}$, Mohammad Anis ${ }^{1}$, Abdulrahman A. Alatar ${ }^{2}$, Eslam M. Abdel-Salam ${ }^{2}$ and \\ Mohammad Faisal $^{2^{*}}$ \\ ${ }^{1}$ Plant Biotechnology Laboratory, Department of Botany, Aligarh Muslim University, Aligarh-202 002, India \\ ${ }^{2}$ Department of Botany \& Microbiology, College of Science, King Saud University, P.O Box 2455, Riyadh 11451, Kingdom of \\ Saudi Arabia
}

*For correspondence: faisalm15@yahoo.com

Received 20 October 2020; Accepted 11 January 2021; Published 25 March 2021

\begin{abstract}
Pterocarpus marsupium Roxb. is a valuable multipurpose forest tree in India. Generally, it is valued greatly for its excellent wood qualities. Due to its significant multipurpose properties, this tree has been overexploited, which ultimately has led to its inclusion in the list of threatened species. In this regard, studying the genetic diversity in P. marsupium is not only significant for the protection of this species, but also necessary for the development and utilization of germplasm resources for its improvement. Before developing any tree improvement program, information on actual genetic diversity and the cryptic number of the differentiated genetic resource are important aids for its conservation and effective utilization. Thus, in the present study, analysis of phylogenetic relationship among $P$. marsupium species plays an important role in the identification and selection of elite genotype among the wildly distributed accessions. The phylogenetic relationship among 18 genotypes obtained from various forest regions of central India was studied using DNA based molecular markers. In RAPD analysis, out of 40 scorable amplified bands, 29 were polymorphic resulting in expression of polymorphism percentage (73.2\%) with an average of 2.90 amplicons per primer. Based on RAPD analysis, the lowest (37\%) similarities among accessions were recorded in Anuppur (MAA), Mandla (MMK) and Jabalpur (MJH) and the highest similarity (100\%) were observed among Mandla (MMK), Jablapur (MJH); Jashpur (CJM), Surguja (CSA), Bilaspur (CBP) and Durg (CDB) and Raigarh (CRK) accessions. While the ISSR analysis found 66 amplified bands, 45 were polymorphous and average 68.3 percent polymorphic with an average 4.5 bands per oligo. The lowest (36\%) similarity was observed among Anuppur (MAA) and Jabalpur (MJH) accessions and the highest similarity $(88 \%)$ was recorded among Jashpur (CJM), Chhindwara (MCD) and Bilaspur (CBP) accessions. The combined analysis data of RAPD and ISSR showed that Chandrapur (RCC) and Anuppur's (MAA) acessions had the lowest (35\%) similarity, with Jabalpur's (MJHs) and Mandla's (MMKs) accession being the highest similarities (100\%) reported. As a result, the study of genetic diversity by means of RAPD and ISSR markers alone or in combination, i.e. the MAA, CKB and CRK accessions, was found to be more diverse among 18 accessions of Central India and given greater space for the collection of elite/superior trees to be used in conservation and forest development programs. (C) 2021 Friends Science Publishers
\end{abstract}

Keywords: Dendrogram; Genetic diversity; Genotype; Molecular markers; Polymorphism

\section{Introduction}

Forest trees provide essential tools for commerce, climate, and industrial production, since these species actively or passively benefit potential society and provide a wide range of sustainable goods and services needed to biodiversity and growth. Herbal remedies in the pharmaceutical industry are greatly in demand and they are conscious that natural medicines are non-toxic and do not have adverse effects. Pterocarpus species are pantropical genus belongs to fabaceae family consisting more of than 70 species worldwide (Saslis-Lagoudakis et al. 2011). In 1972, Rojo reported the main centre for origin of Pterocarpus spp. diversity in tropical forest region of Africa followed by the Indomalaya and Neotropics. Indian Kino tree or Bijasal (Pterocarpus marsupium Roxb.) is a valuable forest tree and known for its herbals importance belonging to the family Fabaceae. The population is abundant in dry mixed deciduous forest and thrives well under modest rainfall of 80-200 cm (NMPB 2008). In India it occupies in central and peninsular region, majorly in forest of Madhya Pradesh, Chhattisgarh, Maharashtra, Andhra Pradesh, some areas of Uttar Pradesh (UP) and sub-Himalayan tracts, up to an altitude of $1000 \mathrm{~m}$. The species can tolerate excessive

To cite this paper: Ahmad A, N Ahmad, M Anis, AA Alatar, EM Abdel-Salam, M Faisal (2021). Molecular fingerprinting for detecting genetic relationships among different accessions of Pterocarpus marsupium. Intl J Agric Biol 25:820-830 
temperature in summer and flourish in deep clayey loam fertile soil with good drainage. Innate species have quickly disappeared, and no new splashes can be seen in the jungle area. It has been listed as endangered species due to autogenic reproductive failure (Barstow 2017). The description of the species in the forest can be characterized by its straight trunk, fissured bark (longitudinal), imparipinnate 5 to 7 leaflets, $8-13 \mathrm{~cm}$ long, coriaceous, dark green and shiny leaves, scented flowers, large panicles with smooth and winged pods. The tree achieved a height of up to $30 \mathrm{~m}$ and a girth of up to $2.5 \mathrm{~m}$ with a straight and transparent bole. The oleo-resin exudates derived from the tree trunk are known as kino-gums. Kino gum is fragrant, brittle, reddish black in color, angular and shiny, and occurs as small flakes.

The tree is a rich source of many active bioconstituents and has gained a lot of interest in recent years in advance study and has been used to treat a variety of diseases since ancient times (Maurya et al. 2004; Hougee et al. 2005; Remsberg et al. 2008; Abirami et al. 2012; Barstow 2017). Now-a-days, it has been studied in modern medicine for its substantial pharmacological properties like antidiabetic drugs (Dhanabal et al. 2006), antimicrobic (Tippani et al. 2010), anti-proliferative and antioxidant (Chakraborty et al. 2010), cardiotonic (Mohire et al. 2007), anti-inflammatory (Hougee et al. 2005; Sander et al. 2005), anthelmintic (Abirami et al. 2012), pain reliever (Tippani et al. 2010), anti-cancer (Remsberg et al. 2008) etc. In addition, the National Medicinal Plant Board (NMPB 2018) of India reports that the annual market capitalization of a tree is approximately 200-500 tons per year in building articles, vessels, bridges, boilers, dividers, and music equipment (Barstow 2017). Due to these listed medicinal and commercial values, $P$. marsupium has gained a great deal of interest in recent years, and the Government of India has begun to promote a campaign for its large-scale production and conservation programmes. The propagating material of the species is only seed, but has a low germination rate (less than 30\%) due to hard fruit coat coupled with a limited viability time and weak pod setting (Anis et al. 2005; Husain et al. 2010; Ahmad et al. 2018). Pathogenic infections on seeds are also affecting their germination rate in natural condition (Yogeshwar et al. 2013). Moreover, it is over-exploited due to its trade in medicinal goods, herbal medicines, local tribal goods, and timber, contributing to its gradual depletion from natural reserves (Chand and Singh 2004; Husain et al. 2010; Ahmad and Anis 2019). In view of restricted distribution, unregulated harvesting, and its inherent qualities, it is important to preserve this precious tree species and to promote ex situ plantation for the production of large-scale plantlets. However, the identification of sustainable or elite genotypes of selected organisms is one of the most critical factors prior to the implementation of any strategic plan for conservation.

The prime aim of the present experimentation was to identify/select elite genotypes of Pterocarpus marsupium among naturally grown accessions through genetic diversity analysis via DNA based molecular markers namely RAPD and ISSR. The RAPD technique uses single primers of random sequences to amplify discrete DNA fragments, which makes it appropriate to reveal polymorphisms among individuals. Whereas ISSR marker supplements the RAPD data as they are generally dominant and detect high level of polymorphism per locus. In combination these markers have been described as the most suitable method for the study genetic diversity in several plant species, as they show high heritability and exhibit maximum polymorphism to discriminate between closely related genotypes even among near isogenic lines (Gupta et al. 2008; Kumar et al. 2009; Kalpana et al. 2012; Dasgupta et al. 2015). Study of genetic variability plays a crucial role in identification and selection of elite genotype among the wildly distributed accessions (Djè et al. 2000; Deshwal et al. 2005; Valadez-Moctezuma et al. 2015). The elite genotype can be utilized for further tree improvement, management of germplasm and evolution of conservation strategies.

\section{Materials and Methods}

\section{Sampling and collection of plant materials}

The accessions of Pterocarpus marsupium have been collected from different central Indian locations and labelled with according to their Global Positioning System (GPS) for future analysis. Number of accessions, locality with their coordinates, and mean girth at breast height $(\mathrm{GBH})$ are described in Table 1. A total of 90 trees were identified phenotypically based on their growth, health and stem types at 18 population sites in the forest regions in central India. Every accession's germplasm, e.g., leaves, was obtained from February to March and each population had around 5 (or by availability) tree accessions of about the same age and a population gap of more than $200 \mathrm{~m}$ between successive accessions in the group. The leaves samples of each accession were collected and immediately packed in ice boxes. Finally, these samples were stored in $-70^{\circ} \mathrm{C}$ deep freezer in the laboratory until processed.

\section{Isolation of genomic DNA}

Genomic DNA was extracted from leaves using modified protocol of Deshmukh et al. (2007). The extraction of DNA was a bit difficult as it has its own restrictions due to occurrence of high content of gummy polysaccharides, polyphenols, and secondary metabolites. Doyle and Doyle (1990) method for DNA extraction was tried several times, but it was found to be impure with yellowish sticky and viscous constituents which acted as inhibitors. By manipulating some few phases of Genomic DNA isolation using the method developed by Deshmukh et al. (2007) in Terminalia arjuna, these inhibitory components were extracted from fresh leaf tissues. 


\section{DNA amplification}

A set of 60 ISSR primers (UBC, Vancouver, BC, Canada) and six set (each having 20) of RAPD primers (OPA, OPB, OPC, OPU, OPV and OPX) kits were used for initial screening. Primer combinations from each (RAPDs and ISSRs) were chosen for assessment of genetic diversity on the primes of PCR amplification sturdiness, scorability and reproducibility of amplicon bands. Reactions for RAPD and ISSR assay were carried out using various amplification reagents and mixtures like 10x buffer with $\left(\mathrm{NH}_{4}\right)_{2} \mathrm{SO}_{4}, 10$ $\mathrm{m} M$ dNTPs mix, $25 \mathrm{mM} \mathrm{MgCl}_{2}$ solution, Taq DNA polymerase (Thermo Scientific, India) and RAPD or ISSR primers. The PCR amplification reactions were conducted in PCR machine (BioRad, U.S.A.). Williams et al. (1990) procedure was followed to carry out the RAPD experiment. RAPD amplification reaction solution $(25 \mu \mathrm{L})$ composed of 10x buffer with $\left(\mathrm{NH}_{4}\right)_{2} \mathrm{SO}_{4}, \mathrm{MgCl}_{2}(25 \mathrm{~m} M)$, dNTPs mixture $(10 \mathrm{~m} M)$, oligos $(10 \mu M)$, Taq polymerase recombinant (2.5 Unit) and DNA template $(50 \mathrm{ng} / \mu \mathrm{L})$. Thermal conditions for PCR amplification was used with initial DNA denaturation for $5 \mathrm{~min}$ at $94^{\circ} \mathrm{C}$ for $5 \mathrm{~min}$ accompanied by 40 cycles including denaturation at $94^{\circ} \mathrm{C}$ for $1 \mathrm{~min}$, annealing at $35^{\circ} \mathrm{C}$ for $1 \mathrm{~min}$ and elongation at $72^{\circ} \mathrm{C}$ for $2 \mathrm{~min}$ ). For $10 \mathrm{~min}$, the final extension was given for $10 \mathrm{~min}$ at $72^{\circ} \mathrm{C}$. In the other hand, ISSR marker technology was applied in line with Zietkiewicz et al. (1994) protocol. Same PCR reaction mixture was used which was optimized in RAPD, except for the annealing temperature $\left(40-60^{\circ} \mathrm{C}\right.$, Table 2). Also, thermo-cycler program for PCR amplification was set same as mentioned in case of RAPD except for 38 reaction cycles. Separation of the amplified DNA products was carried out on $1 \%(w / v)$ agarose gel electrophoresis in using $1 \mathrm{x}$ buffer (TBE) containing $5 \mu \mathrm{g} / \mathrm{ml}$ ETBR (ethidium bromide). The separated DNA bands were scored using a gel documentation system (BioRad, U.S.A.).

\section{Structure analysis}

To examine the population structure of the 18 studied accessions. In this regard, the plateau criterion and $\Delta \mathrm{K}$ methods were applied to analyze the multi-locus genotype data to identify the optimum number of clusters (K) using STRUCTURE v. 2.3 .4 (Pritchard et al. 2000) based on Bayesian model-based and principal component analyses. To identify the optimum K, 10 testing STRUCTURE runs with $1-10 \mathrm{~K}$ value, 10,000 burn-in period and 100,000 Markov Chain Monte Carlo (MCMC) replicates. Admixture model and correlated allele frequencies were assumed. After estimation of the optimum K, 20 STRUCTURE runs with K values from 4 to 6 were performed with the same parameters but 100,000 burn-in period and 750,000 MCMC replicates. The optimum $\mathrm{K}$ was chosen using STRUCTURE HARVESTER website (Earl and vonHoldt 2012). The resulted population and individual Q matrices were then used to visualize the results using DISTRUCT 1.1 (Rosenberg 2004).

\section{Data scoring and analysis}

Well-defined and reproducible bands ranging from 10010,000 bp in both the cases RAPD and ISSR were scored on the basis of presence and absence and documented as 1 and 0 respectively. The size of amplified products was compared by ladder DNA marker (GeneRuler ${ }^{\mathrm{TM}} 10 \mathrm{~kb}$ DNA Ladder). The analyses were based on only distinctly distinct and polymorphic bands and PICs (Polymorphism Information Content) greater than 0.1 were processed to generate similarity matrices. The PIC value of each marker was calculated as $P I C_{i}=2 f_{i}\left(1-f_{i}\right)$. Where $\left(1-f_{i}\right)$ is the frequency of null allele and $f_{i}$ is the frequency of the amplified allele (Roldán-Ruiz et al. 2000). For each first mixture, however, PIC was averaged above the DNA fragments. Using Jaccard 's system of coefficient analysis, the binary matrix used to determine genetic similarity index (Jaccard 1908). The Unweighted Pair Group Method with Arithmetic Mean (UPGMA) for the construction of phonetic dendrogram was subjected to the similarity matrix. Each dendrogram was determined by using NTSYS (Version 2.20) to measure the cophenetic coefficient between the genetic similarities and the cophenetic values matrix (Rohlf 2000). The degree of relationship between the two similarity matrices produced by RAPD and ISSR primers were examined using the product-moment correlation (r) based on Mantel Z-value (Mantel 1967) via $\mathrm{R}$ programming language v. 4.0.3.

\section{Results}

\section{RAPD analysis}

Among the various RAPD primers tested, 10 primers of RAPD gave very good polymorphism across all the 18 accessions of $P$. marsupium. The DNA banding profiles obtained from RAPD analysis are depicted in Table 2 . The amplified number of bands per RAPD oligos ranged from 3 to 6 , with an average of 4.0 bands per oligo. Amplified band numbers per primer varied between 3 and 6 with an average of 4.0 bands per primer. A total of 10 RAPD oligos generated no more than 40 polymorphic bands, 29 of which resulted in $73.2 \%$ of polymorphism and an average of 2.90 polymorphic bands from each primer (Table 3). PIC of 10 RAPD elements were between 0.127 (OPX 19) and 0.170 (OPU 18) with an average PIC value of 10 tested primers as 0.139 (Table 2 and 3). The primer OPU 18 showed maximum percentage of polymorphism $(100 \%)$, whereas the primer OPB 09 exhibited minimum $60 \%$ polymorphism (Table 2). The primer OPA 10 produced highest number of amplified bands (6) whereas four primers (OPA 05, OPU 18, OPV 19 and OPX 03) produced lesser number of amplified bands (3) (Table 2). Fig. 1A-B shows the pattern 
Genetic Diversity of Pterocarpus marsupium / Intl J Agric Biol, Vol 25, No 4, 2021

Table 1: Details of Pterocarpus marsupium Roxb. populations naturally growing in different forest regions of central India

\begin{tabular}{|c|c|c|c|c|c|c|c|}
\hline \multirow[t]{2}{*}{ State } & \multirow[t]{2}{*}{ S.N. } & \multicolumn{4}{|c|}{ Populations } & \multicolumn{2}{|c|}{ Geographical Position } \\
\hline & & District & Forest range/Village & Code & $* \mathrm{GBH}(\mathrm{cm})$ & Latitude $(\mathrm{N})$ & Longitude (E) \\
\hline \multirow[t]{6}{*}{ Madhya Pradesh } & 1 & Mandla & Kalpi Niwas Road & MMK & 125 & $22^{\circ} 55^{\prime} 58^{\prime \prime}$ & $80^{\circ} 12^{\prime} 53^{\prime \prime}$ \\
\hline & 2 & Jabalpur & Hulki & $\mathrm{MJH}$ & 104 & $22^{\circ} 50^{\prime} 52^{\prime \prime}$ & $79^{\circ} 46^{\prime} 16^{\prime \prime}$ \\
\hline & 4 & Hoshangabad & Pipariya & MHP & 130 & $22^{\circ} 39^{\prime} 23^{\prime \prime}$ & $78^{\circ} 23^{\prime} 03^{\prime \prime}$ \\
\hline & 5 & Betule & Gawasen & MBG & 128 & $22^{\circ} 11^{\prime} 18^{\prime \prime}$ & $77^{\circ} 29^{\prime} 01^{\prime \prime}$ \\
\hline & 6 & Seoni & Suktara & MSS & 122 & $21^{\circ} 53^{\prime} 15^{\prime \prime}$ & $79^{\circ} 31^{\prime} 48^{\prime \prime}$ \\
\hline & 7 & Chhindwara & Deogarh & MCD & 138 & $21^{\circ} 52^{\prime} 35^{\prime \prime}$ & $78^{\circ} 43^{\prime} 45^{\prime \prime}$ \\
\hline \multirow[t]{7}{*}{ Chhattisgarh } & 9 & Jashpur & Manora & CJM & 136 & $23^{\circ} 07^{\prime} 26^{\prime \prime}$ & $83^{\circ} 58^{\prime} 24^{\prime \prime}$ \\
\hline & 10 & Surguja & Ambikapur Range & CSA & 133 & $23^{\circ} 03^{\prime} 48^{\prime \prime}$ & $83^{\circ} 17^{\prime} 25^{\prime \prime}$ \\
\hline & 11 & Bilaspur & Pendra Road & CBP & 130 & $22^{\circ} 46^{\prime} 15^{\prime \prime}$ & $81^{\circ} 58^{\prime} 28^{\prime \prime}$ \\
\hline & 12 & Raigarh & Kharsia & CRK & 128 & $22^{\circ} 23^{\prime} 25^{\prime \prime}$ & $84^{\circ} 21^{\prime} 44^{\prime \prime}$ \\
\hline & 13 & Korba & Kudmura & CKK & 142 & $22^{\circ} 19^{\prime} 35^{\prime \prime}$ & $83^{\circ} 04^{\prime} 03^{\prime \prime}$ \\
\hline & 14 & Durg & Balod Range & $\mathrm{CDB}$ & 115 & $20^{\circ} 37^{\prime} 11^{\prime \prime}$ & $81^{\circ} 17^{\prime} 04^{\prime \prime}$ \\
\hline & 15 & Kanker & Bhanupratappur & CKB & 126 & $20^{\circ} 16^{\prime} 32^{\prime \prime}$ & $81^{\circ} 03^{\prime} 33^{\prime \prime}$ \\
\hline Maharashtra & 16 & Nagpur & Garra & RNG & 134 & $21^{\circ} 39^{\prime} 40^{\prime \prime}$ & $79^{\circ} 24^{\prime} 36^{\prime \prime}$ \\
\hline Uttar Pradesh & 18 & Lalitpur & Dhorra Range & ULD & 128 & $24^{\circ} 26^{\prime} 59^{\prime \prime}$ & $78^{\circ} 18^{\prime} 44^{\prime \prime}$ \\
\hline
\end{tabular}

* GBH= Girth at breast height; Values of GBH represents mean of five randomly selected trees of each population

Table 2: Details of 20 primers and amplified DNA bands were obtained among 18 accessions of Pterocarpus marsupium

\begin{tabular}{|c|c|c|c|c|c|c|c|}
\hline Sr. No. & Primers & $* \mathrm{AT}\left({ }^{\circ} \mathrm{C}\right)$ & $\begin{array}{l}\text { Total number of bands } \\
\text { amplified }\end{array}$ & No. of polymorphic bands amplified & No. of monomorphic bands amplified & Polymorphism (\%) & **PIC \\
\hline & RAPD & & & & & & \\
\hline 1 & OPA 05 & 37.0 & 3 & 2 & 1 & 66.7 & 0.134 \\
\hline 2 & OPA 10 & 37.0 & 6 & 4 & 2 & 66.7 & 0.130 \\
\hline 3 & OPB 09 & 37.0 & 5 & 3 & 2 & 60.0 & 0.135 \\
\hline 4 & OPB 15 & 37.0 & 4 & 3 & 1 & 75.0 & 0.148 \\
\hline 5 & OPC 02 & 37.0 & 5 & 4 & 1 & 80.0 & 0.136 \\
\hline 6 & OPC 03 & 37.0 & 4 & 3 & 1 & 75.0 & 0.155 \\
\hline 7 & OPU 18 & 37.0 & 3 & 3 & 0 & 100.0 & 0.170 \\
\hline 8 & OPV 19 & 37.0 & 3 & 2 & 1 & 66.7 & 0.129 \\
\hline 9 & OPX 03 & 37.0 & 3 & 2 & 1 & 66.7 & 0.128 \\
\hline 10 & $\begin{array}{l}\text { OPX } 19 \\
\text { ISSR }\end{array}$ & 37.0 & 4 & 3 & 1 & 75.0 & 0.127 \\
\hline 11 & UBC 814 & 41.7 & 9 & 5 & 4 & 55.6 & 0.114 \\
\hline 12 & UBC 818 & 36.3 & 5 & 3 & 2 & 60.0 & 0.121 \\
\hline 13 & UBC 825 & 50.1 & 8 & 5 & 3 & 62.5 & 0.136 \\
\hline 14 & UBC 827 & 54.6 & 6 & 4 & 2 & 66.7 & 0.149 \\
\hline 15 & UBC 834 & 44.5 & 5 & 3 & 2 & 60.0 & 0.131 \\
\hline 16 & UBC 836 & 40.0 & 9 & 7 & 2 & 77.8 & 0.125 \\
\hline 17 & UBC 848 & 39.6 & 8 & 6 & 2 & 75.0 & 0.152 \\
\hline 18 & UBC 855 & 44.5 & 6 & 5 & 1 & 83.3 & 0.145 \\
\hline 19 & UBC 866 & 49.4 & 6 & 4 & 2 & 66.7 & 0.119 \\
\hline 20 & UBC 880 & 44.5 & 4 & 3 & 1 & 75.0 & 0.165 \\
\hline
\end{tabular}

*AT= Annealing temperature; **PIC= Polymorphism information content

of RAPD fingerprinting generated by the PCR amplifications of OPA 10 and OPU 18, respectively. The size of bands varied between 250 to $3000 \mathrm{bp}$. The PCR amplification using RAPD primers gave rise to reproducible amplification products. Based on RAPD analysis, the highest similarity (100\%) of P. marsupium accessions were observed among Mandla (MMK) and Jablapur (MJH); Jashpur (CJM), Surguja (CSA) and Bilaspur (CBP); Durg (CDB) and Raigarh (CRK) accessions. Whereas, the lowest (37\%) similarities were observed among three accessions i.e., Anuppur (MAA), Mandla (MMK) and Jabalpur (MJH) (Table 4). The 18 accessions were categorized in four major clusters, with the Jaccard Cluster Similarity Coefficient ranging from 0.37 to 1.0 , based on the UPGMA (Table 4). The dendrogram showed 4 major clusters - I, II, III and IV each with seven, three, three and five accessions, respectively (Fig. 2). There are two sub-clusters (Ia and Ib) in cluster-I. Sub-cluster Ia having two accessions in same group belongs to Madhya Pradesh (MMK and MJH). The $I b$ sub-cluster contains 5 accessions, of which 2 accessions are in different groups from Madhya Pradesh (MCD and MBS) and 3 accessions in the same group from Chhattisgarh (CJM, CSA and CBP). Two subclusters IIa and IIb were found in cluster-II. Sub-cluster IIa has two accessions from Maharashtra (RNG and RCC) while, IIb has only one accession from Madhya Pradesh (MBG). Cluster III is made up of 3 accessions which can be again sub-divided into two sub-clusters (IIIa and IIIb). The sub-cluster IIIa contains two accessions one each from Uttar Pradesh and Madhya Pradesh (ULD and MSS) while, IIIb has only one accession from Madhya Pradesh (MHP). The cluster IV has again separated in two sub-clusters (IVa and $I V b)$. The sub-cluster 
Table 3: A comparative list showing details of different markers which used to study genetic diversity in Pterocarpus marsupium accessions

\begin{tabular}{llll}
\hline Primer & RAPD & ISSR & RAPD + ISSR \\
\hline Number of Primer used & 10 & 10 & 20 \\
Total number of bands & 40 & 66 & 106 \\
Total number of polymorphic bands & 29 & 45 & 74 \\
Total number of monomorphic bands & 11 & 21 & 32 \\
Percentage of polymorphism (\%) & 73.2 & 68.3 & 69.8 \\
Average number of bands per primer & 4.0 & 6.6 & 5.3 \\
Average number of polymorphic bands per primer & 2.9 & 4.5 & 3.7 \\
Average polymorphism information content (PIC) & 0.139 & 0.136 & 0.138 \\
\hline
\end{tabular}

Table 4: Jaccard's similarity coefficient matrix among P. marsupium accessions based on RAPD markers

\begin{tabular}{|c|c|c|c|c|c|c|c|c|c|c|c|c|c|c|c|c|c|c|}
\hline & MMK & MJH & ULD & MHP & MBG & MSS & MCD & MBS & CJM & CSA & CBP & CDB & MAA & CKK & CKB & RNG & RCC & CRK \\
\hline MMK & 1.00 & & & & & & & & & & & & & & & & & \\
\hline MJH & 1.00 & 1.00 & & & & & & & & & & & & & & & & \\
\hline ULD & 0.77 & 0.77 & 1.00 & & & & & & & & & & & & & & & \\
\hline MHP & 0.85 & 0.85 & 0.81 & 1.00 & & & & & & & & & & & & & & \\
\hline MBG & 0.81 & 0.81 & 0.70 & 0.76 & 1.00 & & & & & & & & & & & & & \\
\hline MSS & 0.81 & 0.81 & 0.95 & 0.85 & 0.73 & 1.00 & & & & & & & & & & & & \\
\hline MCD & 0.82 & 0.82 & 0.86 & 0.86 & 0.82 & 0.82 & 1.00 & & & & & & & & & & & \\
\hline MBS & 0.86 & 0.86 & 0.82 & 0.81 & 0.86 & 0.77 & 0.95 & 1.00 & & & & & & & & & & \\
\hline CJM & 0.91 & 0.91 & 0.78 & 0.77 & 0.82 & 0.74 & 0.91 & 0.95 & 1.00 & & & & & & & & & \\
\hline CSA & 0.91 & 0.91 & 0.78 & 0.77 & 0.82 & 0.74 & 0.91 & 0.95 & 1.00 & 1.00 & & & & & & & & \\
\hline CBP & 0.91 & 0.91 & 0.78 & 0.77 & 0.82 & 0.74 & 0.91 & 0.95 & 1.00 & 1.00 & 1.00 & & & & & & & \\
\hline CDB & 0.42 & 0.42 & 0.41 & 0.44 & 0.48 & 0.42 & 0.44 & 0.46 & 0.44 & 0.44 & 0.44 & 1.00 & & & & & & \\
\hline MAA & 0.37 & 0.37 & 0.41 & 0.44 & 0.42 & 0.42 & 0.44 & 0.41 & 0.39 & 0.39 & 0.39 & 0.80 & 1.00 & & & & & \\
\hline CKK & 0.39 & 0.39 & 0.43 & 0.46 & 0.44 & 0.44 & 0.46 & 0.43 & 0.41 & 0.41 & 0.41 & 0.90 & 0.90 & 1.00 & & & & \\
\hline CKB & 0.44 & 0.44 & 0.43 & 0.46 & 0.50 & 0.44 & 0.46 & 0.48 & 0.46 & 0.46 & 0.46 & 0.90 & 0.81 & 0.91 & 1.00 & & & \\
\hline RNG & 0.82 & 0.82 & 0.78 & 0.70 & 0.74 & 0.74 & 0.75 & 0.78 & 0.83 & 0.83 & 0.83 & 0.44 & 0.39 & 0.41 & 0.46 & 1.00 & & \\
\hline RCC & 0.86 & 0.86 & 0.75 & 0.74 & 0.78 & 0.71 & 0.79 & 0.83 & 0.87 & 0.87 & 0.87 & 0.43 & 0.38 & 0.40 & 0.45 & 0.96 & 1.00 & \\
\hline CRK & 0.42 & 0.42 & 0.41 & 0.44 & 0.48 & 0.42 & 0.44 & 0.46 & 0.44 & 0.44 & 0.44 & 1.00 & 0.80 & 0.90 & 0.90 & 0.44 & 0.43 & 1.00 \\
\hline
\end{tabular}
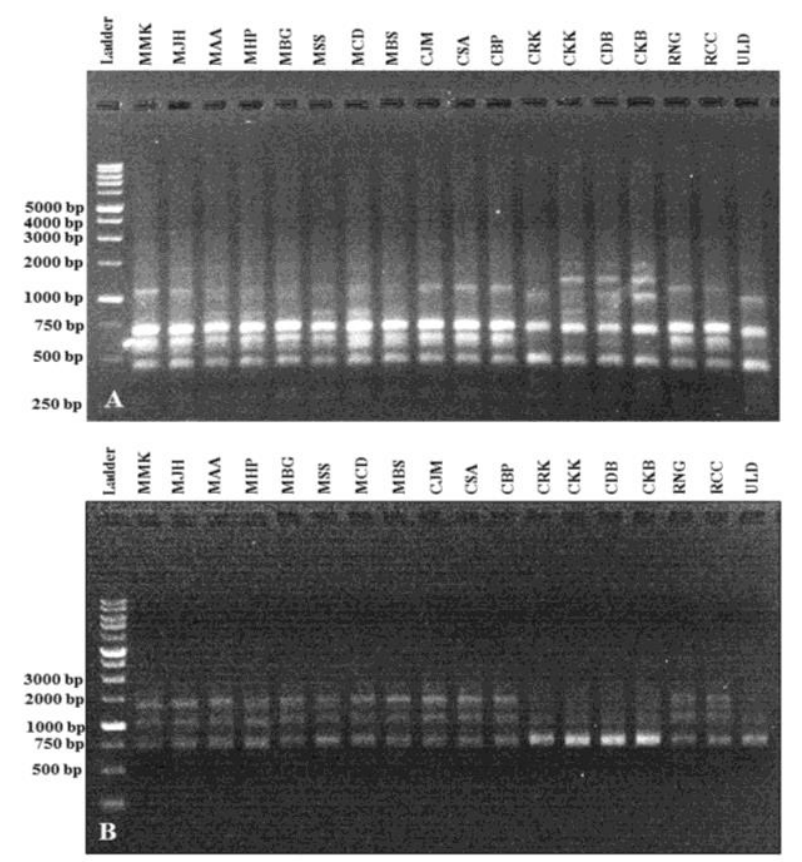

Fig. 1: Representing RAPD banding pattern in different accessions of Pterocarpus marsupium (A) OPA 10; (B) OPB 18

IVa contains 4 accessions from Chhattisgarh (CDB, CRK, $\mathrm{CKK}$ and $\mathrm{CKB}$ ). The sub-cluster $I V b$ is unique as it contains only one accession from Madhya Pradesh (MAA). On the basis of RAPD analysis, sub-cluster $I V b$ consisted MAA accession was found to be more diverse among the eighteen accessions (Fig. 2).

\section{ISSR analysis}

Out of 60 primers screened, 25 primers generated a very clear and separate amplified amplicons, of which 14 oligos exhibited polymorphisms. Finally, 10 best primers used for DNA amplification gave good polymorphism across 18 accessions. The detail of banding profiles of amplified DNA obtained from ISSR markers are shown in Table 2. The number of PCR-amplified DNA products per ISSR-oligos varied between 4 and 9 with an average of 6.6 bands per primer (Table 2). In the first place, a total of 66 bands were amplified, of which 45 were polymorphic, showing an average $68.3 \%$ polymorphic bands, with an average of 4.5 per oligo, while the other 21 bands had monomorphic bands with an average of 2.1 per oligo (Table 3 ). 10 ISSR primers were given polymorphism information content (PIC) of between 0.114 (UBC 814) and 0.165 (UBC 880) with an average PIC content of 0.136 for ten primers (Table 2 and 3). Primer UBC 855 showed maximum percentage of polymorphism $(83.3 \%)$, whereas the minimum percentage of polymorphism (55.6\%) was exhibited by UBC 814 primer (Table 2). Two primers namely UBC 814 and UBC 836 produced highest number of nine amplified bands, whereas primer UBC 880 produced lesser number of four 
Genetic Diversity of Pterocarpus marsupium / Intl J Agric Biol, Vol 25, No 4, 2021

Table 5: Jaccard's similarity coefficient matrix among $P$. marsupium accessions based on ISSR markers data

\begin{tabular}{|c|c|c|c|c|c|c|c|c|c|c|c|c|c|c|c|c|c|c|}
\hline & MMK & MJH & ULD & MHP & MBG & MSS & MCD & MBS & CJM & CSA & CBP & CDB & MAA & CKK & CKB & RNG & RCC & CRK \\
\hline MMK & 1.00 & & & & & & & & & & & & & & & & & \\
\hline MJH & 0.86 & 1.00 & & & & & & & & & & & & & & & & \\
\hline ULD & 0.79 & 0.83 & 1.00 & & & & & & & & & & & & & & & \\
\hline MHP & 0.86 & 0.81 & 0.82 & 1.00 & & & & & & & & & & & & & & \\
\hline MBG & 0.74 & 0.77 & 0.71 & 0.76 & 1.00 & & & & & & & & & & & & & \\
\hline MSS & 0.75 & 0.71 & 0.87 & 0.77 & 0.67 & 1.00 & & & & & & & & & & & & \\
\hline MCD & 0.79 & 0.83 & 0.83 & 0.82 & 0.86 & 0.72 & 1.00 & & & & & & & & & & & \\
\hline MBS & 0.75 & 0.86 & 0.79 & 0.77 & 0.82 & 0.68 & 0.87 & 1.00 & & & & & & & & & & \\
\hline CJM & 0.76 & 0.87 & 0.73 & 0.71 & 0.83 & 0.69 & 0.88 & 0.83 & 1.00 & & & & & & & & & \\
\hline CSA & 0.79 & 0.83 & 0.76 & 0.67 & 0.71 & 0.65 & 0.76 & 0.79 & 0.80 & 1.00 & & & & & & & & \\
\hline CBP & 0.77 & 0.80 & 0.74 & 0.72 & 0.76 & 0.64 & 0.88 & 0.84 & 0.85 & 0.74 & 1.00 & & & & & & & \\
\hline CDB & 0.48 & 0.44 & 0.41 & 0.48 & 0.52 & 0.48 & 0.52 & 0.54 & 0.56 & 0.52 & 0.57 & 1.00 & & & & & & \\
\hline MAA & 0.44 & 0.36 & 0.43 & 0.44 & 0.42 & 0.50 & 0.48 & 0.44 & 0.46 & 0.43 & 0.54 & 0.76 & 1.00 & & & & & \\
\hline CKK & 0.50 & 0.41 & 0.48 & 0.44 & 0.48 & 0.56 & 0.54 & 0.50 & 0.52 & 0.48 & 0.59 & 0.82 & 0.86 & 1.00 & & & & \\
\hline CKB & 0.52 & 0.48 & 0.45 & 0.52 & 0.56 & 0.46 & 0.56 & 0.58 & 0.59 & 0.56 & 0.61 & 0.86 & 0.73 & 0.78 & 1.00 & & & \\
\hline RNG & 0.68 & 0.78 & 0.72 & 0.70 & 0.74 & 0.68 & 0.79 & 0.83 & 0.76 & 0.65 & 0.77 & 0.54 & 0.44 & 0.50 & 0.52 & 1.00 & & \\
\hline $\mathrm{RCC}$ & 0.76 & 0.87 & 0.80 & 0.71 & 0.75 & 0.69 & 0.80 & 0.76 & 0.84 & 0.80 & 0.78 & 0.45 & 0.37 & 0.42 & 0.48 & 0.83 & 1.00 & \\
\hline CRK & 0.46 & 0.48 & 0.45 & 0.41 & 0.50 & 0.46 & 0.56 & 0.58 & 0.59 & 0.56 & 0.61 & 0.86 & 0.73 & 0.86 & 0.82 & 0.58 & 0.48 & 1.00 \\
\hline
\end{tabular}

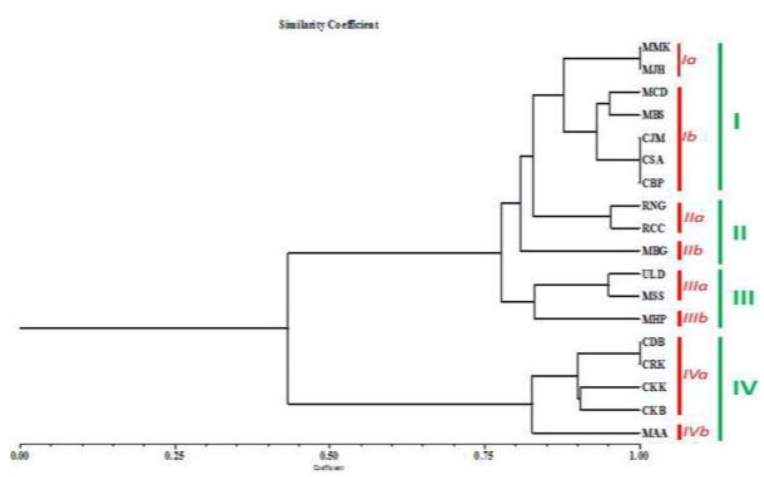

Fig. 2: Dendrogram generated using unweighted pair of group method with arithmetic average analysis, showing genetic relationship among different accessions of Pterocarpus marsupium as revealed from RAPD analysis

amplified bands. The ISSR banding pattern of UBC 825 and UBC 855 are depicted in Fig. 3A-B, respectively. The amplified DNA amplicon size ranged from 250 bp to 3000 bp. On the basis of ISSR analysis, the lowest (36\%) similarity among accessions was observed between Anuppur (MAA) and Jabalpur (MJH), whereas the highest similarity (88\%) was observed among Jashpur (CJM), Chhindwara (MCD) and Bilaspur (CBP) accessions (Table 5). Dendrogram obtained on the basis of UPGMA method through ISSR data is depicted in Fig. 4. The Jaccard coefficient of similarity varied between 0.36 and 0.88 (Table 5). Eighteen accessions were clustered into three major clusters (I, II and III) each with four, nine and five accessions, respectively (Fig. 4). Cluster-I included of two sub-clusters $I a$ and $I b$. Sub-cluster $I a$ consisted of 2 accessions in different groups from Madhya Pradesh (MMK and MHP) and sub-clusters $I b$ comprised of two accessions, of which one belongs to Uttar Pradesh (ULD) and another to Madhya Pradesh (MSS). The Cluster II is sub-clustered in two. Sub-cluster IIa has six accessions, of which three are from Madhya Pradesh (MJH, MCD and MBS), two from

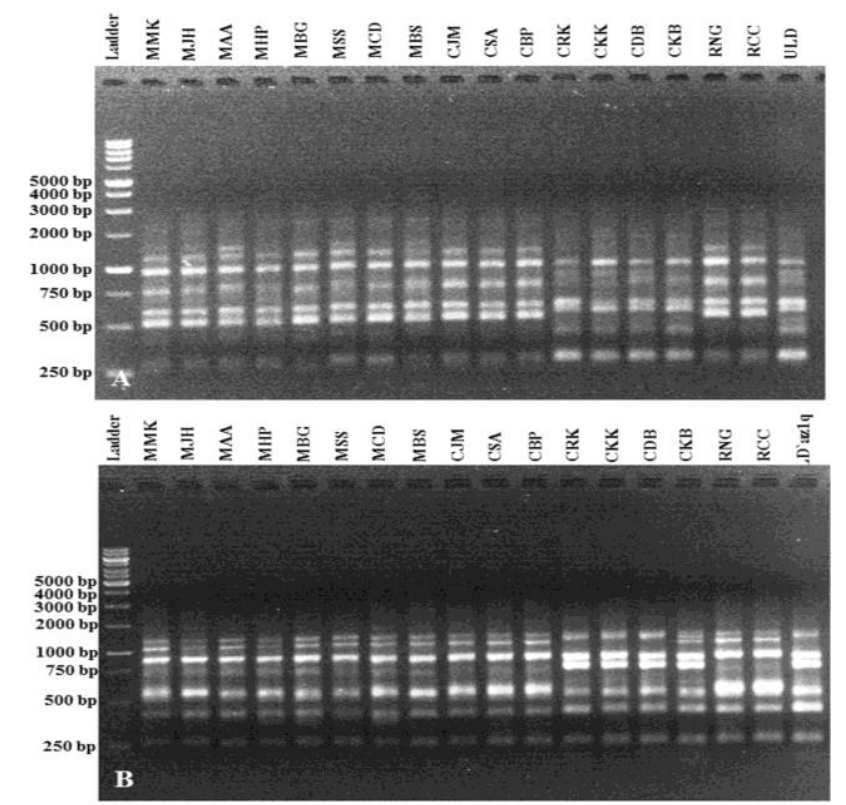

Fig. 3: Representing ISSR banding pattern in different accessions of Pterocarpus marsupium (A) UBC 825; (B) UBC 855

Chhattisgarh (CJM and CBP) and one from Maharashtra (RCC). While sub-cluster $I I b$ consisted of a mixture of three accessions, each from Madhya Pradesh (MBG), Maharashtra (RNG) and Chhattisgarh (CSA). Cluster III has again separated in two sub-clusters which consisted of five accessions. Sub-cluster IIIa containing 4 accessions belongs to Chhattisgarh (CDB and CKB, CKK and CRK). Whereas, sub-cluster $I I I b$ is unique as it contains only one accession from Madhya Pradesh (MAA) and was more diverse among eighteen accessions (Fig. 4).

\section{Population structure}

The Q matrix produced by STRUCTURE analysis showed that the 18 studied accessions clustered into 4 main clusters 


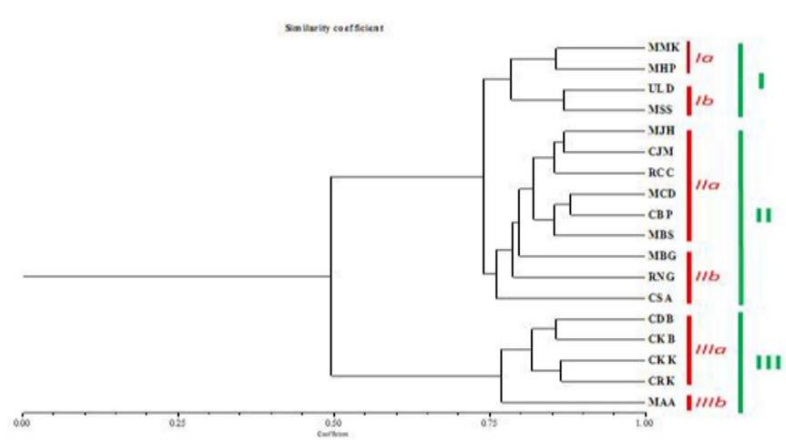

Fig. 4: Dendrogram showing genetic relationship among different accessions of Pterocarpus marsupium as revealed from ISSR analysis

(Fig. 5). One cluster contained 7 accessions (MMK, MJH, MCD, MBS, CJM, CSA, CBP). The Second cluster contained 4 accessions (MBG, ULD, MSS, MHP). The 2 accessions from Maharashtra (RNG) and Chandrapur (RCC) were clustered together. The last cluster contained 5 accessions (CDB, CKK, MAA, CKB, CRK).

\section{Comparative RAPD and ISSR analysis}

The two set of 20 primers (ten each of RAPD and ISSR) gave good polymorphism across all the 18 accessions screened. These primers were produced a total of 106 bands, of which 74 were polymorphic with an average of 5.3 per primer resulting in an average percentage of $69.8 \%$ polymorphism. The average PIC of 20 primers observed was 0.138 (Table 3). An almost-similar clustering pattern for either RAPD or ISSR was created from a dendrogram based on the combined data of RAPD and ISSR, whereas the Jaccard's similarity coefficient ranged from 0.35 to 1.0 (Table 6). The combined analyses showed lowest (35\%) similarity among Chandrapur (RCC) and Anuppur (MAA) accessions, whereas the highest similarities (100\%) were recorded among Jabalpur (MJH) and Mandla (MMK) accessions (Table 6). Four main clusters (I, II , III and IV), with seven, 4, 2 and 5 accessions respectively, were displayed in the Dendrogram based on combined results (Fig. 6). The I-Cluster was divided into two $I a$ and $I b$ subclusters. The subdivision $I a$, which is part of Madhya Pradesh with two accessions into one group (MMK and $\mathrm{MJH})$. The $I b$ sub-cluster contains 5 accessions, of which two are from Madhya Pradesh (MCD and MBS) and 3 from Chhattisgarh (CJM, CSA and CBP). However, cluster II classified into sub-clusters $I I a$ and $I I b$ and sub-cluster IIa has only one accession from Madhya Pradesh (MBG). Subcluster IIb contained 3 accessions, of which 2 were further divided in ULD from Uttar Pradesh and MSS from Madhya Pradesh, whereas only one accession from Madhya Pradesh (MHP). The cluster III has only 2 accessions namely RNG and RCC from Maharashtra which can be subdivided into two sub-clusters IIIa and IIIb, respectively. The cluster IV has again separated into sub-clusters $I V a$ and $I V b$ and $I V a$

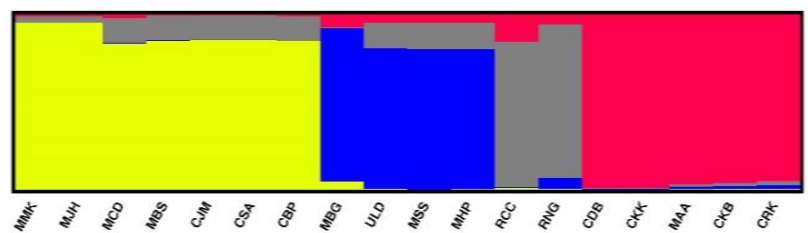

Fig. 5: Population structure analysis of the 18 studied Pterocarpus marsupium accessions as revealed by STRUCTURE analysis. DISTRUCT software was used to visualize the analysis results

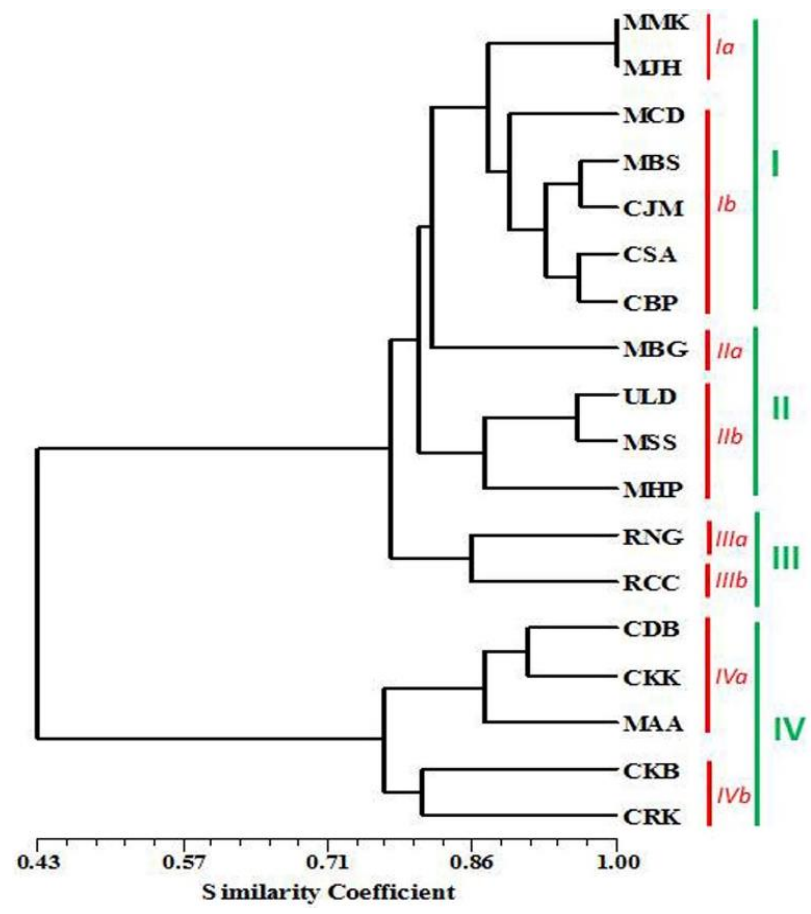

Fig. 6: Dendrogram showing genetic relationship among different accessions of Pterocarpus marsupium as revealed from combined data of RAPD + ISSR analysis

contains 3 accessions, of which 2 are from Chhattisgarh (CDB and CRK) while one from Madhya Pradesh (MAA). Sub-cluster $I V b$ contained only 2 accessions from Chhattisgarh (CKB and CRK). Consequently, these accessions (sub-cluster $I V b$ ) were found to be more diverse among the eighteen accessions analyzed (Fig. 5). The product-moment $(\mathrm{r})$ between similarity matrices produced by RAPD and ISSR primers showed significant positive correlation $(p=0.031, \mathrm{r}=0.651)$.

\section{Discussion}

Forest trees are valuable commodities to the global economy as well as for nature and ecological resources to be protected and restored. Several countries, like India, are having serious problems with preserving these forest resources owing to anthropogenic and recent climate shifts that they are unregulated in their exploitation. This has 
Genetic Diversity of Pterocarpus marsupium / Intl J Agric Biol, Vol 25, No 4, 2021

Table 6: Jaccard's similarity coefficient matrix among $P$. marsupium accessions based on combined data of RAPD and ISSR markers

\begin{tabular}{|c|c|c|c|c|c|c|c|c|c|c|c|c|c|c|c|c|c|c|}
\hline & MMK & MJH & ULD & MHP & MBG & MSS & MCD & MBS & CJM & CSA & CBP & CDB & MAA & CKK & CKB & RNG & RCC & CRK \\
\hline$\overline{\mathrm{MMK}}$ & 1.00 & & & & & & & & & & & & & & & & & \\
\hline MJH & 1.00 & 1.00 & & & & & & & & & & & & & & & & \\
\hline ULD & 0.79 & 0.79 & 1.00 & & & & & & & & & & & & & & & \\
\hline MHP & 0.85 & 0.85 & 0.85 & 1.00 & & & & & & & & & & & & & & \\
\hline MBG & 0.79 & 0.79 & 0.72 & 0.78 & 1.00 & & & & & & & & & & & & & \\
\hline MSS & 0.82 & 0.82 & 0.96 & 0.88 & 0.75 & 1.00 & & & & & & & & & & & & \\
\hline MCD & 0.79 & 0.79 & 0.86 & 0.85 & 0.85 & 0.82 & 1.00 & & & & & & & & & & & \\
\hline MBS & 0.89 & 0.89 & 0.83 & 0.82 & 0.82 & 0.79 & 0.89 & 1.00 & & & & & & & & & & \\
\hline CJM & 0.93 & 0.93 & 0.80 & 0.79 & 0.79 & 0.77 & 0.86 & 0.96 & 1.00 & & & & & & & & & \\
\hline CSA & 0.89 & 0.89 & 0.83 & 0.82 & 0.82 & 0.79 & 0.89 & 0.93 & 0.96 & 1.00 & & & & & & & & \\
\hline CBP & 0.86 & 0.86 & 0.79 & 0.79 & 0.85 & 0.76 & 0.93 & 0.89 & 0.93 & 0.96 & 1.00 & & & & & & & \\
\hline $\mathrm{CDB}$ & 0.42 & 0.42 & 0.42 & 0.45 & 0.50 & 0.44 & 0.47 & 0.45 & 0.44 & 0.45 & 0.47 & 1.00 & & & & & & \\
\hline MAA & 0.38 & 0.38 & 0.42 & 0.45 & 0.45 & 0.44 & 0.47 & 0.41 & 0.40 & 0.41 & 0.42 & 0.83 & 1.00 & & & & & \\
\hline CKK & 0.40 & 0.40 & 0.44 & 0.47 & 0.47 & 0.45 & 0.48 & 0.43 & 0.42 & 0.43 & 0.44 & 0.91 & 0.91 & 1.00 & & & & \\
\hline CKB & 0.44 & 0.44 & 0.44 & 0.47 & 0.47 & 0.46 & 0.44 & 0.47 & 0.46 & 0.47 & 0.44 & 0.81 & 0.74 & 0.81 & 1.00 & & & \\
\hline RNG & 0.83 & 0.83 & 0.83 & 0.76 & 0.76 & 0.79 & 0.77 & 0.80 & 0.83 & 0.86 & 0.83 & 0.45 & 0.41 & 0.43 & 0.47 & 1.00 & & \\
\hline RCC & 0.76 & 0.76 & 0.70 & 0.69 & 0.75 & 0.67 & 0.76 & 0.73 & 0.77 & 0.79 & 0.82 & 0.39 & 0.35 & 0.37 & 0.38 & 0.86 & 1.00 & \\
\hline CRK & 0. & 0.38 & 38 & 0.41 & 0.41 & 0.39 & 0.38 & 0.41 & 0.40 & 0.41 & 0.38 & 0.83 & 0.68 & 0.76 & 0.81 & 0.41 & 0.39 & 1.00 \\
\hline
\end{tabular}

contributed to a major reduction in the number of important plant species. However, unfortunately, because of its continual over-use by many organizations Pterocarpus marsupium has now reached the verge of disappearance as an endangered tree species (Barstow 2017). For the conservation and stopping over-exploitation, there is an urgent need to encourage the ex situ plantation, that requires large scale plantation materials. For this purpose, a simple, cost effective and quick protocol for propagation is essential for achieving practical goal towards conservation and sustainable use. Plant tissue culture is a technique of growing plant cells, tissues and organs in an artificially prepared nutrient medium under aseptic conditions. The technique plays a major role in conservation of the germplasm, rapid clonal propagation, and regeneration of genetically manipulated super clones for ex-situ conservation of valuable trees. However, before developing any regeneration protocol one of the most important aspects is the identification of elite/superior genotypes of desired species. Thus, we have chosen phenotypically healthy tree populations of $P$. marsupium from different areas of central India on the basis of the abundance of their population for purpose of genetic diversity analysis followed by in vitro propagation of selected genotype.

Genetic diversity analysis of $P$. marsupium accessions is beneficial for future management and their conservation. Therefore, it is vital to evaluate how genetic information in natural populations may vary across different geographic and climatic areas by molecular markers. One critical application of molecular markers is to assess the genetic variation in the context of conservation. In most cases, anthropogenic impacts have led to the decline of taxa, whether through overharvesting, habitat destruction, or more recently, changes in climate. To develop an effective strategy to protect, promote, and maintain genetic diversity, it must first be quantified. The genetic data provide relevant information for identifying units of conservation and illuminate the genetic processes that take place in the populations such as patterns of genetic flux, bottlenecks, and genetic drift. Therefore, genetic diversity analysis of $P$. marsupium will be effectively use for selection of superior population for breeding programme, aimed at improving productivity, wood quality and chemical constituents, and also help in future plans for conservation and sustainable use of this valuable plant species. Several PCR-based high-performance technologies, like RAPD and ISSR, RFLP and SSR, have been developed during the couple of decades for testing DNA-level genetic polymorphism. Of these, the RAPD technique uses single primers with random sequences to amplify discrete DNA fragments, which makes it appropriate to reveal polymorphisms among individuals. Whereas ISSR marker supplements the RAPD data as they are generally dominant and detect high level of polymorphism per locus. Both, RAPD and ISSR have been used increasingly to study a large number of plant species in depth in their genetics (Amri and Mamboya 2012; Zhang et al. 2013; Bal et al. 2014; Dasgupta et al. 2015; Anerao et al. 2016; Tiwari et al. 2016; Bajpe et al. 2018). The target sequences of both (RAPD and ISSR) primers are found in more frequently throughout the genome and amplified quickly, which consequently helps reveal a much larger number of polymorphic loci than other dominant markers. In the present study, eighteen accessions of $P$. marsupium from different locations of central India were characterized by using two molecular signs such as RAPD and ISSR, and both the marker were consistent and showed strong genetic diversity among the accessions growing in MAA (Anuppur), CKB (Kanker), CRK (Raigarh), RNG (Nagpur) and RCC (Chandrapur) regions of central India. In a given population, polymorphism is mostly attributed to the presence of genetic variations identified by the number of alleles on a locus and their population distribution frequency. RAPD has extensively been used for detection of 
genetic diversity analysis in several naturally grown tropical forest tree species (Degen et al. 2001), Jatropha curcas (Gupta et al. 2008), Prunus armeniaca (Kumar et al. 2009), Pterocarpus angolensis (Amri and Mamboya 2012), Morus alba (Kalpana et al. 2012), Pterocarpus species (Bal et al. 2014), Bruguiera gymnorrhiza and Haritiera fomes (Dasgupta et al. 2015) and Garcinia xanthochymus (Anerao et al. 2016). Besides, researchers also utilized ISSR genetic diversity identification strategies in a wide range of plant species including mulberries (Awasthi et al. 2004), Hagenia abyssinica (Feyissa et al. 2007), Momordica charantia (Behera et al. 2008), apricot genotypes (Kumar et al. 2009), Pongamia pinnata (Kesari et al. 2010), Tectona grandis (Ansari et al. 2012), Butea monosperma (Vashishtha et al. 2013), Larix gmelinii (Zhang et al. 2013), Quercus brantii (Alikhani et al. 2014), Neolamarckia cadamba (Yiing et al. 2014), Andrographis paniculata (Tiwari et al. 2016), Mimosa caesalpiniaefolia (dos Santos Araújo et al. 2016) and Salacia species (Bajpe et al. 2018).

Comparative analysis of the 2 types of molecular markers indicated that the level of polymorphism depends on the size and inter-and intra-specific diversity of the samples (Gupta et al. 2008). In this study, RAPD primers were found to be more efficient than ISSR as there was $73.2 \%$ polymorphism among the accessions compared to $68.3 \%$ by ISSR. The findings are different from the results achieved for many other plant species (Ajibade et al. 2000; Costa et al. 2016). A total of 20 primers (RAPD + ISSR) yielded 74 polymorphic bands among the 18 accessions grouped into four clusters. Jaccard's similarity coefficientbased RAPD data showed that genetic similarity ranged from 100\% (MJH-MMK, CBP-CJM-CSA, CDB-CRK) to $37 \%$ (MMK-MAA-MJH) among different accessions. The UPGMA based analysis grouped all the eighteen accessions in different four clusters (I, II, III and IV) with seven, three, three and five accessions in each group. However, the cumulative number of polymorphic and discriminating fragments for ISSR is greater than that for RAPD. Zietkiewicz et al. (1994) documented that ISSR-PCR analysis have high potential to reveal polymorphism and provide a significant potential for intergenome variation relative to other random primers such as RAPD. Genetic variations accumulate as geographically isolated colonies adapt to different landscapes. While genetic similarity was between 36\% (MAA-MJH) and 88\% (CJM-MCD-CBP) among the accessions in the ISSR analysis. Eighteen accessions were divided into 3 clusters (I, II and III), each with four, nine and five accessions respectively. These findings are consistent with several similar scientific reports on some other higher plants available (Gupta et al. 2008; Bal et al. 2014; Sharma et al. 2014). All dendrograms showed a similar total topology with only few differences for the majority of accessions. Accessions could be classified according to the data obtained into four clusters for RAPD and RAPD + ISSR, while the ISSR data analysis based dendrogram showed 3 clusters. The dendrogram based on combined data showed 4 clusters each having seven, four, two and five accessions respectively. This result was further confirmed by the $\mathrm{Q}$ matrix produced by the population structure analysis as the 18 studied accessions were clustered into 4 main clusters with the same accessions in each cluster. Our results are in accordance with several other finding in different plants (Kumar et al. 2009; Sharma et al. 2014; Valadez-Moctezuma et al. 2015). Meanwhile, the findings obtained may be used as a preliminary step for further researches on the population structure and developmental genetics of certain genotypes. Furthermore, the analysis will certainly help to detect elite and superior accessions by identifying degrees of intra and inter-specific genetic variation within the $P$. marsupium populations.

\section{Conclusion}

Based on individual or combined RAPD and ISSR data analysis, MAA, CKB and CRK accessions were found to be more diverse among the genotypes. These accessions offer better possibilities to select candidates plus trees for functional diversity, such as better seed germination, seedling growth and biochemical contents, due to the high genetic diversity. The RAPD and ISSR molecular analyzes gave us valuable knowledge about the phylogenetic linkage between the various accessions and provided that both these markers may be an essential tool in the generation of diagnostic fingerprints for breeders. The phylogenetic analysis on the based on the derived dendrogram of the marker also supports the fact that there are regional specific variations that can be made prior to the introduction of multiple generations of selection. In the analysis carried out for both RAPD and ISSR, four classes were identified that correspond to the $18 P$. marsupium collections sites of the Central India. Overall, genetic diversity analysis of $P$. marsupium will be effectively use for selection of elite/superior population for breeding program, aimed at improving productivity, wood quality and chemical constituents, and also help in future plans for conservation and sustainable use of this valuable plant species. Furthermore, there is a need to conserve the species for the benefit of mankind. More importantly, critical elements of effective conservation strategies need to be studied.

\section{Acknowledgements}

The authors are thankful to the Research Supporting Project number (RSP-2020/86), King Saud University, Riyadh, Saudi Arabia.

\section{Author Contributions}

AA designed and performed the experiments; NA, AAA, EMA and MF gave input in experimental design, demonstrated the molecular studies, statistical analysis 
and illustrations; MA and MF supervised the experiments; All the authors discussed the results and implications; The manuscript was written by AA and edited by NA, MA and MF

\section{Conflict of Interest}

The authors declare to have no clash of interest among themselves and the institutions where the work was done

\section{Data Availability Declaration}

All data relevant to this article are available with the corresponding author and will be provided on request

\section{References}

Abirami B, P Gayathri, D Uma (2012). In vitro antioxidant potential of Pterocarpus marsupium bark. Intl J Chem Pharm Sci 3:17-24

Ahmad A, M Anis (2019). Meta-topolin improves in vitro morphogenesis, rhizogenesis and biochemical analysis in Pterocarpus marsupium Roxb.: A potential drug-yielding tree. J Plant Growth Regul 38:1007-1016

Ahmad A, N Ahmad, M Anis (2018). Preconditioning of nodal explants in thidiazuron-supplemented liquid media improves shoot multiplication in Pterocarpus marsupium (Roxb.). In: Thidiazuron: From Urea Derivative to Plant Growth Regulator, pp:175-187. Ahmad N, M Faisal (Eds.). Springer Singapore, Singapore

Ajibade SR, NF Weeden, SM Chite (2000). Inter simple sequence repeat analysis of genetic relationships in the genus Vigna. Euphytica 111:47-55

Alikhani L, MS Rahmani, N Shabanian, H Badakhshan, A Khadivi-Khub (2014). Genetic variability and structure of Quercus brantii assessed by ISSR, IRAP and SCoT markers. Gene 552:176-183

Amri E, F Mamboya (2012). Genetic diversity in Pterocarpus angolensis populations detected by random amplified polymorphic DNA markers. Intl J plant Breed Genet 6:105-114

Anerao JS, V Jha, L Korgaonkar (2016). Dissecting genetic diversity in Garcinia xanthochymus using ISSR and RAPD markers. $J$ Plant Breed Genet 4:69-76

Anis M, MK Husain, A Shahzad (2005). In vitro plantlet regeneration of Pterocarpus marsupium Roxb., an endangered leguminous tree. Curr Sci 88:861-863

Ansari SA, C Narayanan, SA Wali, R Kumar, N Shukla, SK Rahangdale (2012). ISSR markers for analysis of molecular diversity and genetic structure of Indian teak (Tectona grandis Lf) populations. Ann For Res 55:11-23

Awasthi AK, GM Nagaraja, GV Naik, S Kanginakudru, K Thangavelu, J Nagaraju (2004). Genetic diversity and relationships in mulberry (genus Morus) as revealed by RAPD and ISSR marker assays. BMC Genet 5; Article 1

Bajpe SN, TR Bharathi, KM Marulasiddaswamy, KKS Kumara, HS Prakash, RK Kini (2018). Efficiency of RAPD, ISSR and ITS markers in detecting genetic variability among Salacia species sampled from the Western Ghats of Karnataka. Mol Biol Rep 45:931-941

Bal P, P Panda, U Mohapatra (2014). Genetic diversity and phylogenetic relationships in Pterocarpus species and its closely related genus Tipuana (Fabaceae) as revealed by RAPD and ISSR markers. Plant Sci Res 36:68-76

Barstow M (2017). Pterocarpus Marsupium. The IUCN Red List of Threatened Species 2017:e.T34620A67802995

Behera TK, AK Singh, JE Staub (2008). Comparative analysis of genetic diversity in Indian bitter gourd (Momordica charantia L.) using RAPD and ISSR markers for developing crop improvement strategies. Sci Hortic 115:209-217
Chakraborty A, N Gupta, K Ghosh, P Roy (2010). In vitro evaluation of the cytotoxic, anti-proliferative and anti-oxidant properties of pterostilbene isolated from Pterocarpus marsupium. Toxicol In Vitro 24:1215-1228

Chand S, AK Singh (2004). In vitro shoot regeneration from cotyledonary node explants of a multipurpose leguminous tree Pterocarpus marsupium Roxb. In Vitro Cell Dev Biol Plant 40:464-466

Costa R, G Pereira, I Garrido, MM Tavares-de-Sousa, F Espinosa (2016). Comparison of RAPD, ISSR, and AFLP molecular markers to reveal and classify orchardgrass (Dactylis glomerata L.) germplasm variations. PLoS One 11; Article e 0152972

Dasgupta N, P Nandy, C Sengupta, S Das (2015). RAPD and ISSR marker mediated genetic polymorphism of two mangroves Bruguiera gymnorrhiza and Heritiera fomes from Indian Sundarbans in relation to their sustainability. Physiol Mol Biol Plants 21:375-384

Degen B, H Caron, E Bandou, L Maggia, MH Chevallier, A Leveau, A Kremer (2001). Fine-scale spatial genetic structure of eight tropical tree species as analysed by RAPDs. Heredity 87:497-507

Deshmukh VP, PV Thakare, US Chaudhari, PA Gawande (2007). A simple method for isolation of genomic DNA from fresh and dry leaves of Terminalia arjuna (Roxb.) Wight and Arnot. Electr J Biotechnol 10:468-472

Deshwal RPS, R Singh, K Malik, GJ Randhawa (2005). Assessment of genetic diversity and genetic relationships among 29 populations of Azadirachta indica A. Juss. using RAPD markers. Genet Res Crop Evol 52:285-292

Dhanabal S, C Kokate, M Ramanathan, E Kumar, B Suresh (2006). Hypoglycaemic activity of Pterocarpus marsupium Roxb. Phytother Res 20:4-8

Djè Y, M Heuertz, C Lefèbvre, X Vekemans (2000). Assessment of genetic diversity within and among germplasm accessions in cultivated sorghum using microsatellite markers. Theor Appl Genet 100:918-925

dos Santos Araújo F, MV Pacheco, F de Almeida Vieira, C dos Santos Ferrari, FC Félix, KPT das Chagas (2016). ISSR molecular markers for the study of the genetic diversity of Mimosa caesalpiniaefolia Benth. Idesia 34:47-52

Doyle J, J Doyle (1990). Isolation of DNA from small amounts of plant tissues. BRL Focus 12:15

Earl DA, BM vonHoldt (2012). STRUCTURE HARVESTER: A website and program for visualizing STRUCTURE output and implementing the Evanno method. Conserv Genet Res 4:359-361

Feyissa T, H Nybom, IV Bartish, M Welander (2007). Analysis of genetic diversity in the endangered tropical tree species Hagenia abyssinica using ISSR markers. Genet Resour Crop Evol 54:947-958

Gupta S, M Srivastava, G Mishra, P Naik, R Chauhan, S Tiwari, M Kumar, R Singh (2008). Analogy of ISSR and RAPD markers for comparative analysis of genetic diversity among different Jatropha curcas genotypes. Afr J Biotechnol 7:4230-4243

Hougee S, J Faber, A Sanders, RBD Jong, WBVD Berg, J Garssen, MA Hoijer, HF Smit (2005). Selective COX-2 inhibition by a Pterocarpus marsupium extract characterized by pterostilbene, and its activity in healthy human volunteers. Planta Med 71:387-392

Husain MK, M Anis, A Shahzad (2010). Somatic embryogenesis and plant regeneration in Pterocarpus marsupium Roxb. Trees 24:781-787

Jaccard P (1908). Nouvelles researchers sur la distribution florale Société. Vaud Sci Nat 44:22-27

Kalpana D, SH Choi, TK Choi, K Senthil, YS Lee (2012). Assessment of genetic diversity among varieties of mulberry using RAPD and ISSR fingerprinting. Sci Hortic 134:79-87

Kesari V, VM Sathyanarayana, A Parida, L Rangan (2010). Molecular marker-based characterization in candidate plus trees of Pongamia pinnata, a potential biodiesel legume. AoB Plants 2010; Article plq017

Kumar M, GP Mishra, R Singh, J Kumar, PK Naik, SB Singh (2009). Correspondence of ISSR and RAPD markers for comparative analysis of genetic diversity among different apricot genotypes from cold arid deserts of trans-Himalayas. Physiol Mol Biol Plants 15:225-236 
Mantel N (1967). The detection of disease clustering and a generalized regression approach. Cancer Res 27:209-220

Maurya R, R Singh, M Deepak, SS Handa, PP Yadav, PK Mishra (2004). Constituents of Pterocarpus marsupium: An ayurvedic crude drug. Phytochemistry 65:915-920

Mohire NC, VR Salunkhe, SB Bhise, AV Yadav (2007). Cardiotonic activity of aqueous extract of heartwood of Pterocarpus marsupium. Ind J Exp Biol 45:532-537

NMPB (2008). National Medicinal Plant Board, Department of AYUSH, Ministry of Health and Family Welfare, Government of India, Agro techniques of selected medicinal plants. TERI press, New Delhi, India

NMPB (2018). National medicinal plant board of india. Available at: http://nmpb.nic.in/medicinal_list

Pritchard JK, M Stephens, P Donnelly (2000). Inference of population structure using multilocus genotype data. Genetics 155:945-959

Remsberg CM, JA Yáñez, Y Ohgami, KR Vega-Villa, AM Rimando, NM Davies (2008). Pharmacometrics of pterostilbene: Preclinical pharmacokinetics and metabolism, anticancer, antiinflammatory, antioxidant and analgesic activity. Phytother Res 22:169-179

Rohlf F (2000). NTSYS-pc: Numerical taxonomy and multivariate analysis system, Version 2.2. Exeter Software, Setauket, New York, USA

Roldán-Ruiz I, J Dendauw, EV Bockstaele, A Depicker, MD Loose (2000). AFLP markers reveal high polymorphic rates in ryegrasses (Lolium spp.). Mol Breed 6:125-134

Rosenberg NA (2004). distruct: A program for the graphical display of population structure. Mol Ecol Notes 4:137-138

Sander S, H Smit, J Garssen, J Faber, M Hoijer (2005). Pterocarpus marsupium extract exhibits antiinflammatory activity in human subjects. Plant Med 71:387-392

Saslis-Lagoudakis CH, BB Klitgaard, F Forest, L Francis, V Savolainen, EM Williamson, JA Hawkins (2011). The use of phylogeny to interpret cross-cultural patterns in plant use and guide medicinal plant discovery: An example from Pterocarpus (Leguminosae). PLoS One 6; Article e22275
Sharma SS, K Aadil, MS Negi, S Tripathi (2014). Efficacy of two dominant marker systems, ISSR and TE-AFLP for assessment of genetic diversity in biodiesel species Pongamia pinnata. Curr Sci 106: $1576-1580$

Tippani R, M Porika, V Allenki, RNR Anreddy, NR Yellu, DR Krishna, C Thammidala, S Abbagani (2010). Antioxidant and analgesic activities of Pterocarpus marsupium Roxb. J Herbs Spices Med Plants 16:63-68

Tiwari G, R Singh, N Singh, DR Choudhury, R Paliwal, A Kumar, V Gupta (2016). Study of arbitrarily amplified (RAPD and ISSR) and gene targeted (SCoT and CBDP) markers for genetic diversity and population structure in Kalmegh [Andrographis paniculata (Burm. f.) Nees]. Ind Crop Prod 86:1-11

Valadez-Moctezuma E, S Samah, A Luna-Paez (2015). Genetic diversity of Opuntia spp. varieties assessed by classical marker tools (RAPD and ISSR). Plant Syst Evol 301:737-747

Vashishtha A, T Jehan, S Lakhanpaul (2013). Genetic diversity and population structure of Butea monosperma (Lam.) Taub.- a potential medicinal legume tree. Physiol Mol Biol Plants 19:389-397

Williams JG, AR Kubelik, KJ Livak, JA Rafalski, SV Tingey (1990). DNA polymorphisms amplified by arbitrary primers are useful as genetic markers. Nucl Acids Res 18:6531-6535

Yiing TS, CS Fu, HW Seng, PS Ling (2014). Genetic diversity of Neolamarckia cadamba using dominant DNA markers based on inter-simple sequence repeats (ISSRs) in Sarawak. Adv Appl Sci Res 5:458-463

Yogeshwar M, R Rimi, N Brajesh, S Fatima (2013). Effect of seed orientation and medium strength on In vitro germination of Pterocarpus marsupium Roxb. Not Sci Biol 5:476-479

Zhang L, HG Zhang, XF Li (2013). Analysis of genetic diversity in Larix gmelinii (Pinaceae) with RAPD and ISSR markers. Genet Mol Res 12:196-207

Zietkiewicz E, A Rafalski, D Labuda (1994). Genome fingerprinting by simple sequence repeat (SSR)-anchored polymerase chain reaction amplification. Genomics 20:176-183 